\title{
Attention Systems and the Organization of the Human Parietal Cortex
}

\author{
M. F. S. Rushworth, ${ }^{1,2}$ T. Paus, ${ }^{3}$ and P. K. Sipila ${ }^{3}$ \\ ${ }^{1}$ Department of Experimental Psychology, Oxford University, Oxford, OX1 3UD, United Kingdom, ${ }^{2}$ Oxford Centre for \\ Functional Magnetic Resonance Imaging of the Brain, Department of Clinical Neurology, Oxford University, John Radcliffe \\ Hospital, Oxford, OX3 9DU, United Kingdom, and ${ }^{3}$ Montreal Neurological Institute, Montreal, Quebec, H3A 2B4 Canada
}

Event-related functional magnetic resonance imaging was used to compare activity in the human parietal cortex in two attention-switching paradigms. On each trial of the visual switching (VS) paradigm, subjects attended to one of two visual stimuli on the basis of either their color or shape. Trials were presented in blocks interleaved with cues instructing subjects to either continue attending to the currently relevant dimension or to switch to the other stimulus dimension. In the response switching (RS) paradigm, subjects made one of two manual responses to the single stimulus presented on each trial. The rules for stimulus-response mapping were reversed on different trials. Trials were presented in blocks interleaved with cues that instructed subjects to either switch stimulus-response map- ping rules or to continue with the current rule. Brain activity at "switch" and "stay" events was compared. The results revealed distinct parietal areas concerned with visual attentional set shifts (VS) and visuomotor intentional set shifts (RS). In VS, activity was recorded in the lateral part of the intraparietal region. In $\mathrm{RS}$, activity was recorded in the posterior medial intraparietal region and adjacent posterior superior and dorsomedial parietal cortex. The results also suggest that the basic functional organization of the intraparietal sulcus and surrounding regions is similar in both macaque and human species.

Key words: LIP; MIP; PE; conditional motor selection; intraparietal sulcus; set-switching
There is no definite consensus on the correspondence between monkey and human parietal cortex. Brodmann (1909) argued that, in the monkey, the superior parietal lobule (SPL) and inferior parietal lobule (IPL) consist principally of areas 5 and 7, respectively. In the human, however, he suggested that both areas 5 and 7 are in the SPL, whereas new areas 39 and 40 are found in the IPL. Other anatomists, however, have identified a correspondence between the cytoarchitecture of the human and macaque IPL and the human and macaque SPL (Von Bonin and Bailey, 1947; Eidelburg and Galaburda, 1984). According to this view, the IPL and SPL are organized similarly in both species, and the intraparietal sulcus divides the parietal cortex similarly in both species.

Functional imaging has not resolved this debate. Such studies suggest that visuospatial attention is correlated with posterior parietal activity in human subjects, but different studies locate the critical region in the SPL or IPL, and some even suggest that an extensive region including both lobules is associated with visuospatial attention (Corbetta et al., 1993, 1995; Nobre et al., 1997; Coull and Nobre, 1998; Corbetta and Shulman, 1999; Gitelman et al., 1999). It is therefore not clear if the human parietal cortex has

\footnotetext{
Received Jan. 24, 2001; revised April 30, 2001; accepted April 30, 2001.

This work was supported by the Royal Society, the Medical Research Council (United Kingdom), the Canadian Institutes of Health Research, and the Canadian Foundation for Innovation. We gratefully acknowledge the advice of K. Worsley, C. Liao, V. Petre, B. Pike, and H. Johansen-Berg.

Correspondence should be addressed to Matthew F. S. Rushworth, Department of Experimental Psychology, University of Oxford, Oxford, OX1 3UD, UK. E-mail: matthew.rushworth@psy.ox.ac.uk.

Copyright (C) 2001 Society for Neuroscience $\quad 0270-6474 / 01 / 215262-10 \$ 15.00 / 0$
}

a similar or dissimilar functional organization to that of the macaque, in which visuospatial attention and oculomotor control depend on the posterior IPL and adjacent lateral intraparietal sulcus (Andersen et al., 1998; Colby and Goldberg, 1999).

In studies of visual attention, subjects are usually required to respond with hand movements, although visual attention is more naturally linked with eye movements than hand movements. The neuroimaging studies may have inadvertently activated not just a system for visual attention but also an attentional system related to hand movements; there is evidence that the lateral and medial banks of the intraparietal sulcus of the macaque are concerned with visual attention-eye movements and with hand movements, respectively (Colby and Duhamel, 1991; Snyder et al., 1997, 1998; Eskander and Assad, 1999). Previous human neuroimaging studies may have activated the human homologs of both areas.

In the present experiments we compared two task-switching paradigms. In both paradigms, event-related functional magnetic resonance imaging (fMRI) was used to measure brain activity at the time of a task switch. In the visual switch (VS) paradigm, subjects switched between attending to one of two stimuli on the basis of either color or shape. Attending to the shape or color of a stimulus means that subjects also attend to its spatial location (Tsal and Lavie, 1988, 1993). Manual responses were only rarely required at the time of attentional switching. The response switch (RS) paradigm required a switch of visuomotor-related intention; subjects switched between selecting one of two responses, cued by visual stimuli, according to one of two rules. Switch-related activity was recorded in the lateral intraparietal region in VS but in the medial intraparietal region and adjacent posterior SPL in RS. The results suggest that similar functional regions are located in the medial and lateral intraparietal sulcus in both humans and monkeys. 


\section{MATERIALS AND METHODS}

\section{Subjects}

A total of 20 right-handed, healthy volunteers participated in the fMRI recording study (ages 19-31 years). The vision of all subjects was normal or corrected to normal with MRI-compatible glasses. Ten subjects performed the RS paradigm, and 10 performed the VS paradigm. The data from two subjects who performed the VS task was lost after main power failures disrupted data acquisition and storage. All subjects gave their informed consent before participation. The procedures were approved by the Research Ethics Committee of the Montreal Neurological Institute and Hospital. Different subjects participated in each experiment because sufficient data could only be gathered for one paradigm in a given scanning session. Moreover, in this way, the potential for task confusion was avoided; because of matching, the cues used in the two tasks were identical in appearance but dissimilar in terms of their behavioral role.

\section{Behavioral tasks}

Both paradigms were conducted with subjects lying in the dimly illuminated MRI scanner. Stimuli were projected onto a screen using a liquid crystal display projector at the head of the scanner tube. Subjects wore prism goggles so that the stimuli appeared directly in front of them. We have previously shown that attentional or intentional task switching is associated with a similar behavioral cost, measured in terms of reaction time, in both VS and RS paradigms (M. F. S. Rushworth, A. C. Nobre, and R. E. Passingham, unpublished observations).

Response switching. Figure 1 (left-hand column) summarizes the RS task. The RS task targeted the mechanisms of attention or task-switching related to the execution of manual responses. On each trial, subjects saw either a red triangle $\left(5.1^{\circ}\right.$ width, $2.7^{\circ}$ height $)$ or square $\left(3.7^{\circ}\right.$ width, $2.7^{\circ}$ height). During the first set of trials, subjects made a right-hand response to the square and a left-hand response to the triangle. A small circle $\left(0.9^{\circ}\right.$ diameter, $70 \mathrm{msec}$ duration) provided feedback to the subjects $100 \mathrm{msec}$ after the response (yellow for correct responses and blue for incorrect responses). An interval of $800 \mathrm{msec}$ followed before the onset of the next trial. The intervals between trial onsets varied according with reaction time and averaged $\sim 1500 \mathrm{msec}$.

Each experimental session was broken down into blocks of 9-11 trials. The rules by which responses were selected varied between blocks; on some blocks subjects responded to squares and triangles by pressing buttons with the left and right hands, respectively. Each block was preceded by an instruction cue. Instruction cues were either a vertical $(+)$ or a tilted $(\mathrm{x})$ cross appearing in a white rectangular background $\left(6^{\circ}\right.$ width, $5^{\circ}$ height) presented for $200 \mathrm{msec}$. Cues indicated that the subject should either switch rules for response selection or stay with the current response selection rules. There was a $1000 \mathrm{msec}$ interval between the onset of the instructive cue and the onset of the first pair of items. The meaning assignment (switch, stay) of each cue $(x,+)$ was counterbalanced across subjects.

Trials were presented in four sessions each of 5 min duration. The event-related analysis was centered on a comparison of BOLD signal after the switch and stay cues. The timing of both types of cue onset was recorded with respect to the onset of acquisition of each frame of fMRI data (see below). A control "rest" cue was presented every $3 \mathrm{sec}$ for the final $25 \mathrm{sec}$ of each session. The rest cue resembled the other instruction cues but consisted of a black circle on a white background. No trials followed the rest cues, and subjects were required to take no action in response to them. No analysis of the rest cues is reported.

Visual switching. Figure 1 (right-hand column) summarizes the VS task. The VS paradigm complemented the RS paradigm and shared most aspects of its formal design. It was designed to study the mechanisms of visual attentional task switching. Subjects were required to switch attention between different sensory dimensions of stimuli. Two visual stimulus items were presented simultaneously ( $70 \mathrm{msec}$ duration) to either side $\left(1.7^{\circ}\right.$ eccentricity) of a white central fixation cross $\left(1.3^{\circ}\right.$ width, $1.1^{\circ}$ height $)$ on a black background. The two items always consisted of one square shape $\left(1.7^{\circ}\right.$ width $\times 2^{\circ}$ height $)$ and one triangular shape $\left[2.6^{\circ}\right.$ width (narrower at base), $2^{\circ}$ height]. One of the items was always green, and one of the items was always red. Either shape could be combined with either color. Subjects used either a particular shape (e.g., square) or color (e.g., red) to direct their attention to the relevant item to detect embedded targets (see below). There was a variable 1200-1500 msec interval between trials.

As in the RS paradigm, each experimental session was broken down into shorter blocks of $9-11$ trials. At the beginning of an experimental

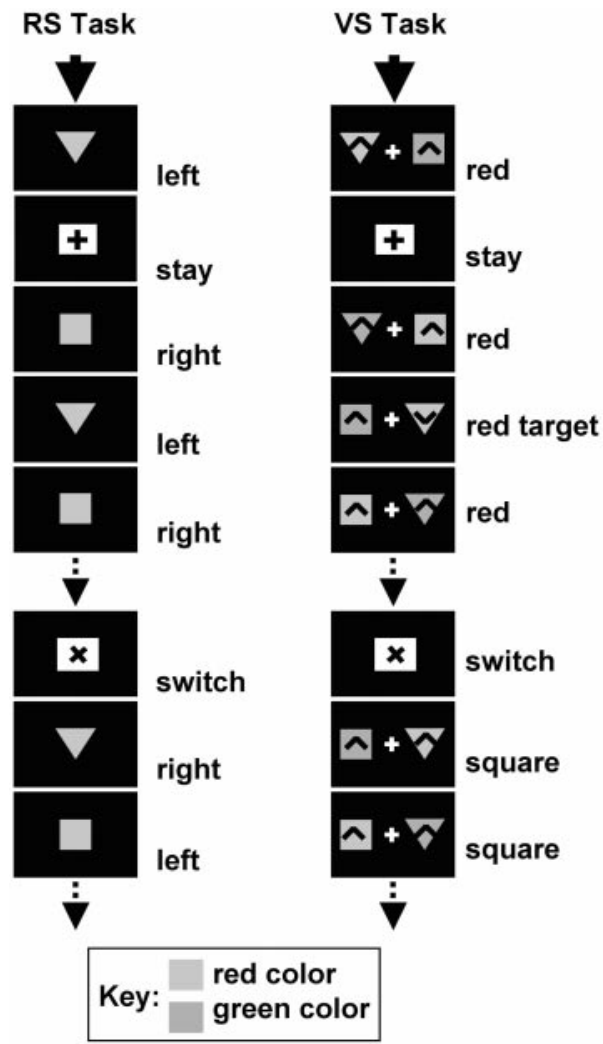

Figure 1. Details of the RS task are shown in the left-hand column. Subjects were presented with a series of task items. The items were always either squares or triangles. Subjects alternated between two response selection rules either triangle-left-hand and square-right-hand or triangle-right-hand and square-left-hand. The figure shows an example in which the subject started with the first rule and later switches to the second rule. Every 9-11 trials, a white cue shape instructed subjects to either stay with the current selection rule set or to switch to using the other rule set. Stay or switch cues were differentiated by a + or $\mathrm{x}$ at their center. The meaning of the + and $\mathrm{x}$ was counterbalanced across subjects. In the example shown, the + and the $\mathrm{x}$ mean stay and switch, respectively. The right-hand column shows examples of stimuli used in the VS task. The task was formally similar to the RS task. On each trial subjects were presented with a pair of stimuli (items) either side of a central fixation point. One of the stimuli was always red, and the other was green. One of the stimuli was always a square, and the other was a triangle. The subject attended to just one of the two stimuli according to a rule based on either color or shape. The design was fully counterbalanced so that some subjects alternated between attending to red or square stimuli, while others alternated between red or triangle, green or square, or green or triangle. For example, the subject might start (top panel) by attending to just the red stimulus item on every trial, regardless of its shape. Every 9-11 trials, a white cue shape instructed subjects to either stay with the current selection rule or to switch to using a selection rule based on the other stimulus dimension. For example, the subject might then attend to the square stimulus regardless of color (seventh panel from top). Again, stay or switch cues were differentiated by a + or $\mathrm{x}$ at their center. The meaning assignments of the + and $\mathrm{x}$ were counterbalanced across subjects as before. In the example shown, the + and the $\mathrm{x}$ mean stay and switch, respectively. The subject's task was to detect a rare target, $V$ ( fourth panel from top), and respond with a key press. The $V$ only ever appeared in the attended stimulus and only on $20 \%$ of trials. On other trials only nontarget $\Lambda$ were presented, and no response was required. Both the target and non-target $V$ and the $\Lambda$ were only present for the final $15 \mathrm{msec}$ of the total $70 \mathrm{msec}$ of stimulus presentation.

block, during the first set of trials, subjects were told to attend to one particular stimulus feature (e.g., red color) and identify targets that appeared within the relevant (red) item. Subsequently, instruction cues appeared before each set of 9-11 trials. Instruction cues were either a 
vertical $(+)$ or a tilted (x) cross appearing in a white rectangular background $\left(6^{\circ}\right.$ width, $5^{\circ}$ height $)$ presented for $200 \mathrm{msec}$. Cues indicated that the subject should switch the current visual rule for selection or stay with the current visual rule. There was a $1000 \mathrm{msec}$ interval between the onset of the instructive cue and the onset of the first pair of items.

The visual selection rule was switched between particular predefined features in the two different dimensions of color and shape. Each subject was instructed to attend to a particular color or a particular shape. For example, a given subject might be instructed to switch between attending to the square shape and the red color while another subject might be asked to switch between attending to the triangle shape and the green color. In this way the task could be made simple for each individual subject, but the relevant version of each dimension could counterbalanced across the group of subjects. So some subjects alternated between red color-square shape, others between green color-triangle shape, others between green color-square shape, and still others between red color-triangle shape.

The sequence of events that would be encountered by a subject switching attention between the red color and the square shape can be summarized. Starting with the relevant feature "red" the switch cue (e.g., $\mathrm{x})$ would inform the subject that the relevant feature became "square." The next switch cue instructed the subject that the relevant feature returned to being "red." The appearance of the stay cue $(+)$ instructed subjects to continue selecting items based on their current visual rule. The meaning assignment (switch, stay) of each cue $(\mathrm{x},+)$ was also counterbalanced across subjects.

The counterbalancing of cue assignment and selection features ensured that behavioral measures were not confounded with artifacts because of different physical appearances of the stimuli. Five levels of matched red and green luminosities were used randomly for item colors throughout the experiment. Differences in the physical intensity of stimuli therefore were unlikely to contribute systematically to attentional effects.

To ensure feature-guided sensory attention, subjects were asked to discriminate small target stimuli embedded within the items. A small $\left(0.7^{\circ}\right.$ long and $\left.0.06^{\circ} \mathrm{high}\right)$ horizontal or angled line was presented in the middle of each item. The embedded stimulus appeared only briefly ( 15 $\mathrm{msec}$ ) at the end of each item presentation (55 msec after item onset) to maximize the advantage of orienting toward the relevant item. On most trials $(80 \%)$, embedded non-targets were presented; the non-target was either a horizontal line or a line angled upward (approximating a "v") to different degrees $\left(0.06^{\circ}\right.$ to $\left.2.9^{\circ}\right)$. On rare $(20 \%)$ target trials, the line was deviated downward (into a "w", always by $2.9^{\circ}$ ). Subjects responded upon the detection of the rare target (w) with a single key-press. Targets (w) only ever appeared in the relevant visual dimension to which subjects were attending.

As for RS, VS trials were presented in four sessions, each of $5 \mathrm{~min}$ duration. The event-related analysis was centered on a comparison of BOLD signal after the switch and stay cues. A control rest cue was presented every $3 \mathrm{sec}$ for the final $25 \mathrm{sec}$ of each session. The rest cue resembled the other instruction cues but consisted of a black circle on a white background. No trials followed the rest cues, and subjects were required to take no action in response to them. No analysis of the rest cue period is reported.

\section{MRI acquisition}

Scanning was performed on a $1.5 \mathrm{~T}$ Siemens Vision magnet. The scanning procedure began with the acquisition of a high-resolution T1 structural anatomical scan [ 80 slices at a thickness of $2 \mathrm{~mm} ; 256 \times 256$ matrix size; repetition time (TR), $22 \mathrm{msec}$; echo time (TE), $10 \mathrm{msec}$; flip angle, $30^{\circ}$; voxel size, $1 \times 1 \times 2 \mathrm{~mm}^{3}$ ]. This was immediately followed by acquisition of four series of 120 gradient-echo images $(20$ slices of $5 \mathrm{~mm}$ thickness in the same orientation as the Sylvian fissure starting above the most dorsal cortex; $64 \times 64$ matrix size; TR, $2.441 \mathrm{msec}$; TE, $50 \mathrm{msec}$; flip angle, $90^{\circ}$; voxel size, $5 \times 5 \times 5 \mathrm{~mm}^{3}$ ) of BOLD signal while subjects performed the behavioral tasks.

\section{Event-related fMRI data analysis}

Each subject's T1 structural image was transformed into standard stereotaxic space (Talairach and Tournoux, 1988) based on 305 brains (Evans et al., 1992) using in-house software (Collins et al., 1994). BOLD signal images were smoothed with a three-dimensional (3-D) $6 \mathrm{~mm}$ (full width half maximum) Gaussian kernel, corrected for head motion artifact and transformed into the same standard stereotaxic space. The statistical analysis was performed with adapted in-house software (Worsley et al.,

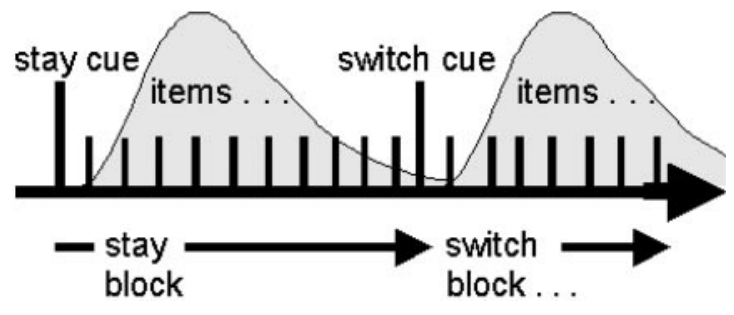

Figure 2. The RS and VS tasks were formally similar. Both tasks were composed of blocks of 9-11 items separated by the presentation of cues. The cues were either switch or stay cues that instructed subjects to switch the selection rule or to continue with the current selection rule, respectively. The BOLD signals, modeled as $\gamma$-density functions with a mean lag of $7 \mathrm{sec}$ and an SD of $3 \mathrm{sec}$ (gray regions) after switch and stay cues were compared.
RS Task

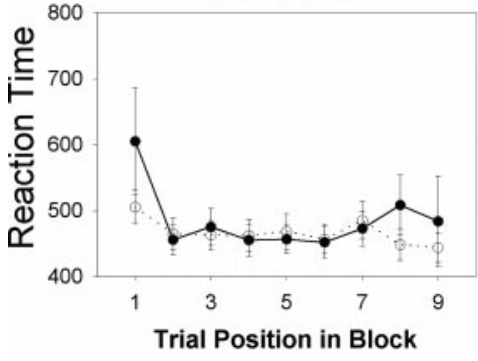

VS Task

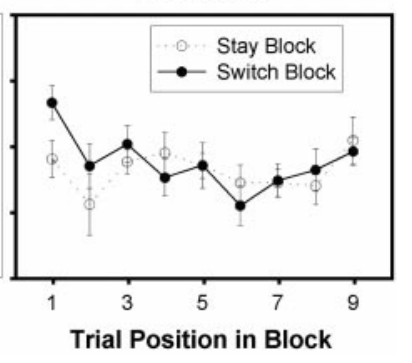

Figure 3. Behavioral results from the VS (left) and RS (right) tasks. The black line shows RTs recorded from switch block trials. The dotted line shows RTs recorded from stay block trials. In both tasks, switching selection rule was associated with an RT cost that was most apparent on the first trial of the block and that subsequently diminished.

2000) using a method based on a linear model with correlated errors and a random effects analysis. Task-related brain activity was measured by examining the BOLD signal after the switch and stay cues in the VS and RS paradigms (Fig. 2); the BOLD signal was convolved with a hemodynamic response function that was modeled as a $\gamma$-density function with a mean lag of $7 \mathrm{sec}$ and a SD of $3 \mathrm{sec}$ (Zarahn et al., 1997) timed to coincide with the onset of switch or stay cues. It has been shown that set shifting is not completed on presentation of an instruction cue (Rogers and Monsell, 1995; Allport and Wylie, 1999; Rushworth et al., 1999). The set shift is only completed after initiation of the next block. The convolved BOLD signal reflects neural processes operating at both the beginning of the set shift (on cue presentation) and at the end of the set shift (on presentation of the first task item). Drift was removed by adding third-order polynomial covariates in the volume acquisition times in the design matrix (which were not convolved with the hemodynamic response function). Random-effects T-statistical maps of significant difference between cue-related BOLD signals were constructed by using a spatially smoothed (15 $\mathrm{mm}$ full width half maximum Gaussian kernel) estimate of the random effects variance. The $t$ statistical maps were then thresholded $(t>4.75 ; p<0.01 ; t>5.19 ; p<0.001)$ in accordance with the Bonferonni correction for multiple comparisons, for the entire 20 slice brain volume-scanned, and non-isotropic random field theory (Worsley et al., 1996, 1999). Although the analysis was performed without previous definition of a region of interest limited to the parietal cortex we report, in detail, just the results for the parietal cortex.

\section{RESULTS}

\section{Behavioral results}

Switching in both VS and RS paradigms was associated with a behavioral cost that could be measured in reaction time (RT). Nine of the 10 RS subjects responded more slowly on the first trial of a switch block than they had on the first trial of a stay block (Figs. 2, 3a). The mean RTs for the first responses in stay and switch RS blocks were 505 and $605 \mathrm{msec}$, respectively (Wilcoxon, 


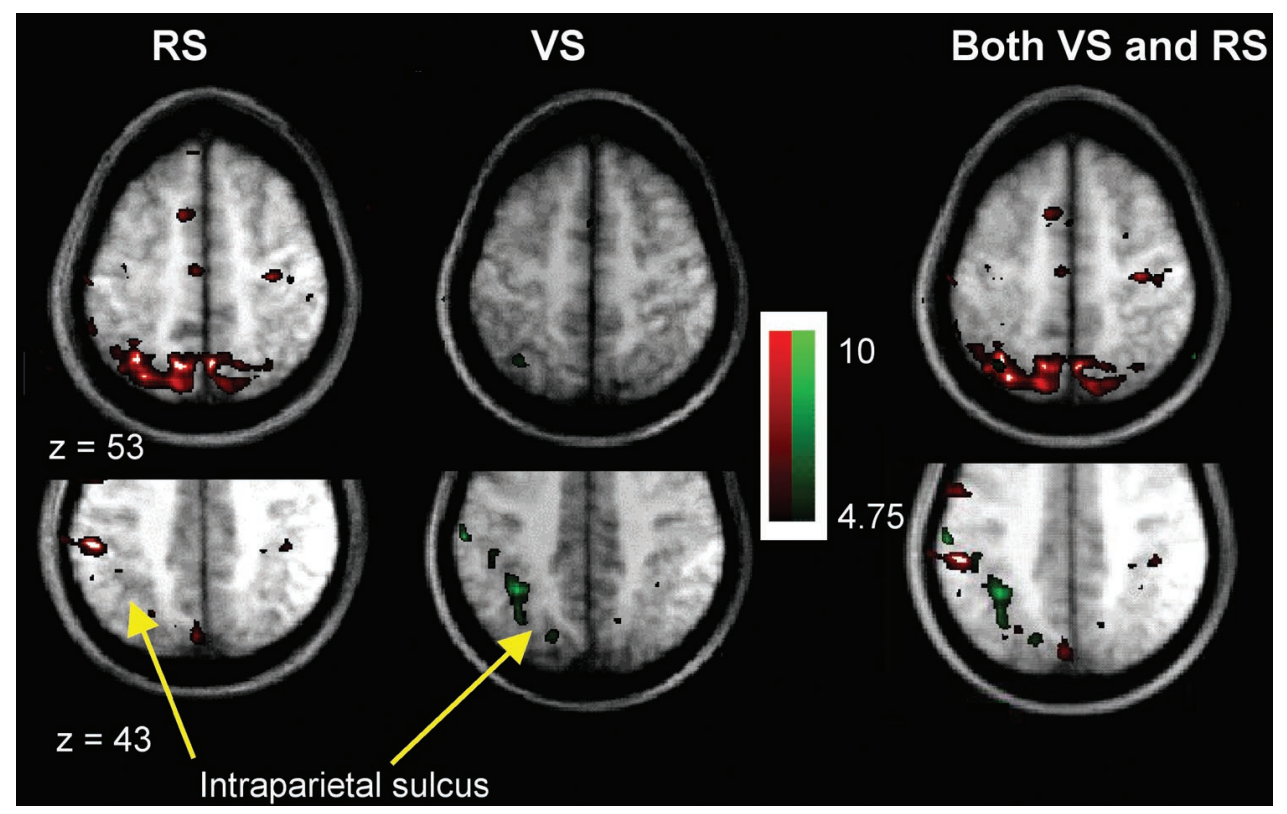

Figure 4. Axial sections through the parietal lobe showing group $t$-statistical maps of significant BOLD signal increase in VS (green) and RS (red) tasks (threshold, $t>$ $4.75 ; p<0.01)$ in the lateral bank of the intraparietal sulcus. The VS and RS results are shown separately on the left and together on the right. Activations are shown superimposed on average brain anatomical MRIs of participating subjects in Talairach and Tournoux (1988) space. The results for both tasks are shown together in the righthand column, superimposed on the average MRI for all participating subjects. VS (green) activity is most prominent in the posterior lateral intraparietal sulcus in the more ventral section $(z=43)$. More anteriorly, in the same section, within the lateral intraparietal sulcus, however, RS (red) activity predominates. The more dorsal section $(z=53)$ shows RS-related activity confined to the medial intraparietal region and the posterior superior parietal lobule.

$t=0 ; N=10 ; \mathrm{p}=0.008)$. All eight VS subjects responded more slowly on the first trial of a switch block than they had on the first trial of a stay block (Fig. 3b). The mean RTs for the first responses in the stay and switch VS blocks were 579 and $665 \mathrm{msec}$, respectively, which differed significantly (Wilcoxon, $t=0 ; N=8 ; p=$ 0.012).

\section{fMRI results}

Switching in both VS and RS paradigms was associated with increases in BOLD signal ("activations") in a number of parietal regions in and around the intraparietal sulcus (Figs. 4-9). VS activations in the parietal cortex were recorded in the lateral bank of the intraparietal sulcus and in the parieto-occipital region. Posterior parietal RS activations were recorded in the medial bank of the intraparietal sulcus and in the adjacent posterior superior and mediodorsal parietal lobule. There were no significant switching-related BOLD signal decreases in the parietal cortex.

More anterior parietal regions, in the supramarginal gyrus and adjacent anterior intraparietal sulcus, were activated mainly in the RS task (Fig. 4). Only one VS task peak, but five RS peaks, were recorded in this region. Figure 4 shows an axial section that approximately follows the intraparietal sulcus. It can be seen that there is a transition from VS- to RS-related activity moving anteriorly along the lateral bank of the intraparietal sulcus.

Both VS and RS tasks activated the more posterior parietal regions, posterior to the supramarginal gyrus. The two tasks, however, activated different regions within the more posterior parietal cortex; RS activations appeared to be on the medial bank of the intraparietal sulcus and the adjacent posterior SPL, whereas VS activations appeared to be on the lateral bank of the intraparietal sulcus. For the most part, the allocation of an activation to one side or other of the intraparietal sulcus is clear. In some cases, because the data are group data, the activations cannot be ascribed with complete certainty to one bank of the intraparietal sulcus or the other. Even in these cases, however, it remains certain that both tasks activate the intraparietal sulcal region and that the VS activations are more ventral and anterior to the RS activations, suggesting that the VS and RS activations are always on the lateral or medial intraparietal sulcal banks, respectively. Viewed in the coronal plane, the intraparietal sulcus lies at $\sim 45^{\circ}$ to the main rostrocaudal and mediolateral axes of the brain (Fig. 5). This means that the lateral and medial banks of the intraparietal sulcus are not distinguished in a simple way by either their relative dorsoventral or mediolateral positions [the conventional Talairach and Tournoux (1988) $y$ and $x$ axes]. The lateral bank is, in general, both ventral and lateral to the medial bank of the intraparietal sulcus. The VS activations were both ventral and lateral to the RS activations. This can be seen in the series of coronal sections (Fig. 5). Moreover, the VS activations in the posterior intraparietal sulcus were rostral to those recorded in the RS paradigm. The more ventral and rostral position of the VS activations with respect to the RS activations can be appreciated in the sagittal section (Fig. 6). In summary, the RS and VS activations around the posterior intraparietal sulcus were differentially localized in all three of the standard brain axes, rostrocaudal, mediolateral, and dorsoventral. That the RS and VS locations are distinct in all three axes makes it difficult to figuratively summarize their relative positions in just a single standard brain section. For this reason RS and VS activations are also shown on a brain section orthogonal to the main axes of the intraparietal sulcus; the differential localization of VS and RS activations are now immediately clear (Fig. 7). The more rostral, lateral, and ventral position of the VS activations compared with the RS activations can also be appreciated in a 3-D view (Fig. 8).

The positions of posterior intraparietal VS and RS activation peaks have been plotted graphically in Figure 9. In this figure all activations have been plotted in the left hemisphere (if an activation peak was at coordinate $16,-66,48$ then it has been plotted at $-16,-66,48)$. Again the separation of the two areas of activation is clear. It can be seen that the separation of VS- and RS-related activations is not simply the artifactual consequence of their existence in different hemispheres.

The positions of VS and RS activation peaks in the parietal cortex are summarized in Table 1. It is clear, once again, that the VS and the RS paradigms are associated with distinct activation clusters. The VS paradigm is associated with activation in the parieto-occipital region (PO) and the lateral intraparietal sulcus (LIP). The RS paradigm is associated with activation in the 


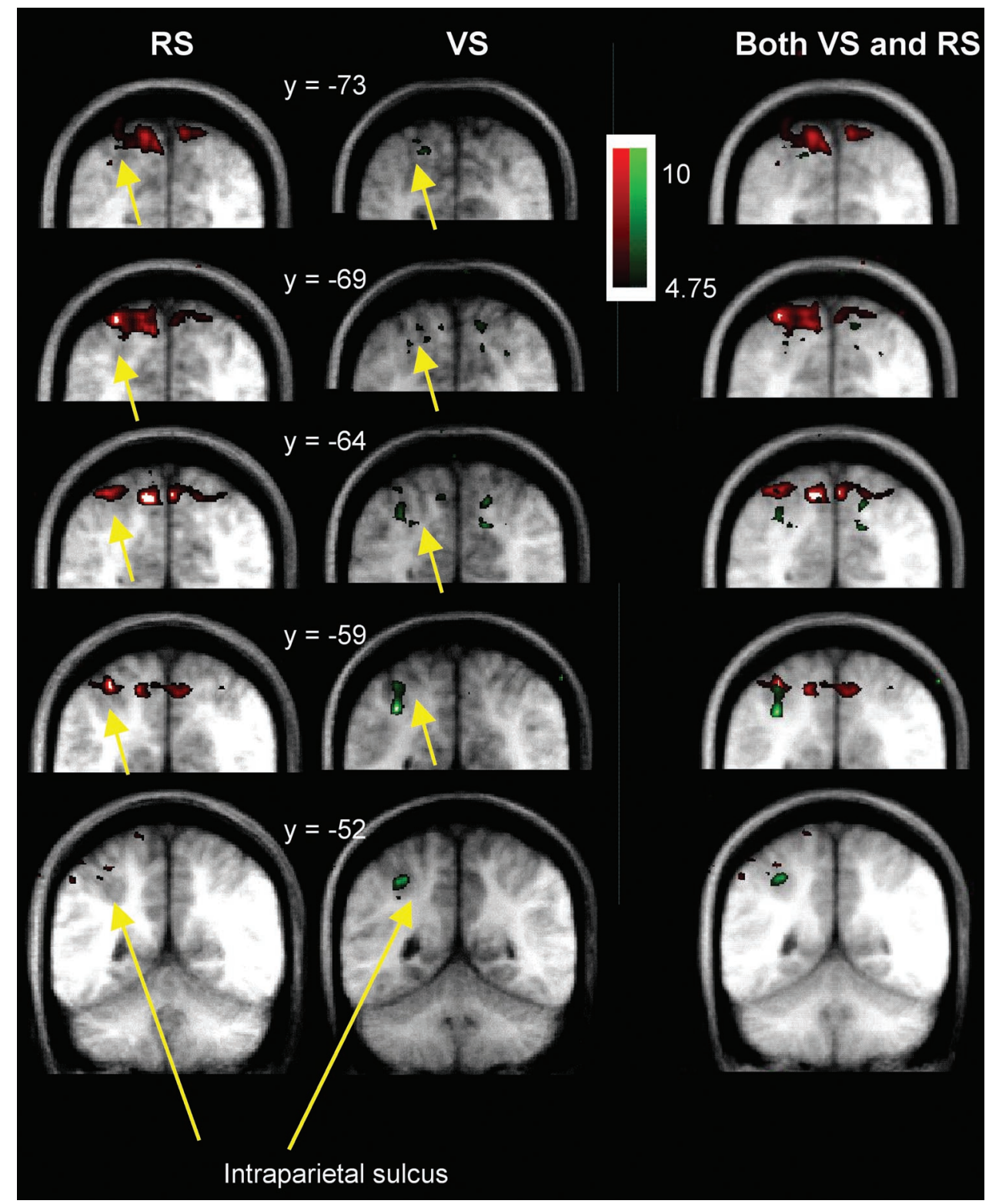

Figure 5. Coronal sections through the parietal lobe showing group $t$-statistical maps of significant BOLD signal increase in the VS (green) and RS (red) tasks. Activations are shown superimposed on average brain anatomical MRIs of participating subjects in Talairach and Tournoux (1988) space. The most posterior section is shown at the bottom, and the most anterior is at the top. The number beside each section gives the position, in millimeters, of the anteroposterior ( $y$-axis) position of the coronal section, with respect to the anterior commissure. Yellow arrows indicate the intraparietal sulcus.

peaks in PO and LIP. The opposite is true in MIP, PEp, and AIP. The third criterion is the relative distribution of activations, produced with each paradigm, with high and low significance values. The significance values of VS activations are higher in LIP and PO than they are in MIP, PEp, and AIP. The significance values of RS activations are higher in MIP, PEp, and AIP than they are in PO and LIP. To test the statistical significance of these differences, we directly compared BOLD signal in the two tasks (i.e., RS switch — stay against VS switch—stay) in a $20,000 \mathrm{~mm}^{3}$ region around the left intraparietal sulcus using the same methods and procedures as the main analyses. We were able to confirm statistically significant greater activation in the RS task than the VS task in the same MIP, PEp, and AIP region that had been identified in the analysis of the simple RS task results (Table 2). We were able to confirm statistically greater activation in the VS task than the RS task in the same LIP region (Table 2) that had been identified in the analysis of the simple VS task results. In addition the VS task, compared with the RS task, was associated with statistically greater activity in a region just anterior and dorsal to the main region of LIP activation previously identified the highest significance values in a given area; the significance values of VS peaks are higher than the significance values of RS action between coordinate position and task $(F=4.212$; df $=2$, $16 ; p=0.034)$, showing that the two tasks were associated with activations in statistically separable regions of the posterior parietal cortex.

The second criterion is which task produces activations with 


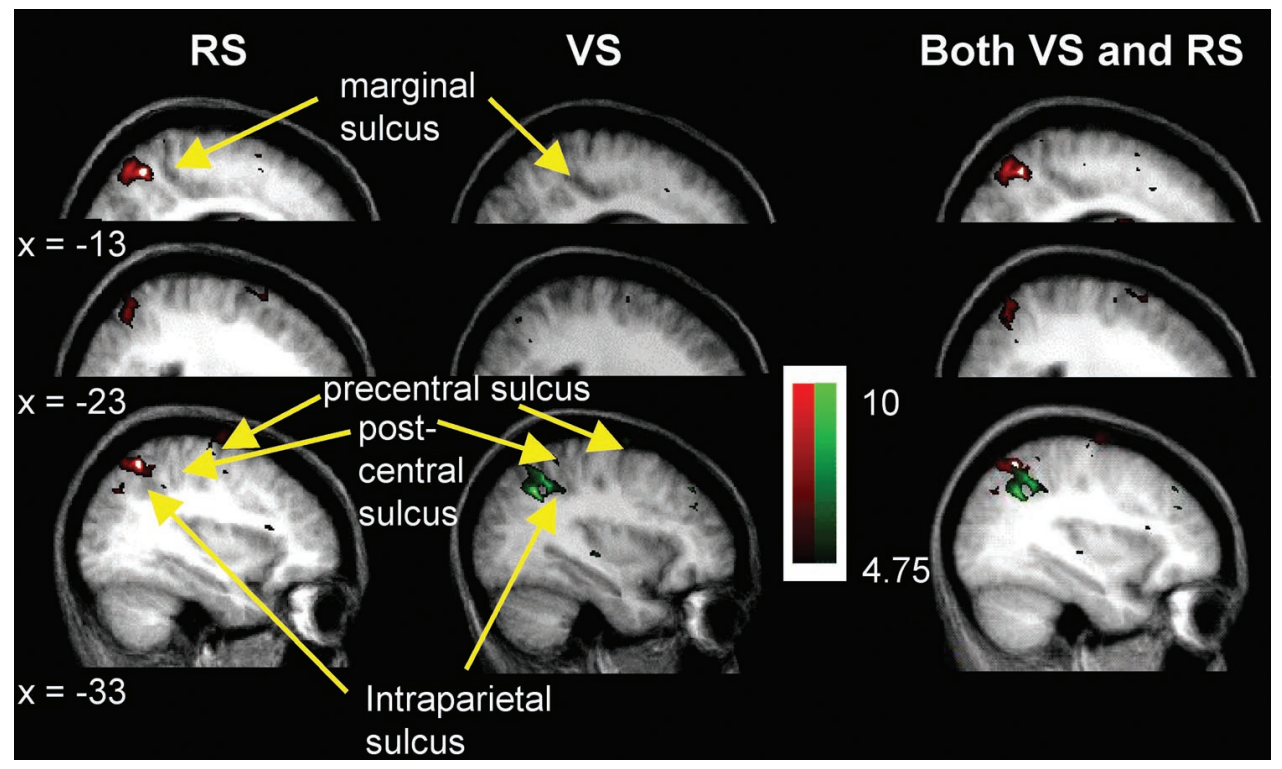

Figure 6. Sagittal sections through the parietal lobe showing areas of group $t$-statistical maps of significant BOLD signal increase in the VS (green) and RS (red) tasks. Activations are shown superimposed on the average brain anatomical MRIs of participating subjects.
$(-30,-45,54)$. The anterior and dorsal shift of this activation probably reflects not just the presence of VS-related activity in this region but the absence of RS related activity here. The location of this activation reflects the fact that the comparison between tasks was not conducted after masking by the main effect of either original RS or VS analysis (which would entail prejudging the task separation being tested) or the task general main of effect of switching (which might preclude the identification of an area strongly associated with one task but not at all associated with another task).

\section{DISCUSSION}

Event-related fMRI was used to compare visual attentional and visuomotor intentional task-switching paradigms, VS and RS. In both cases, subjects' responses were significantly slower on the first trial after a switch. Switching in VS was associated with activation in the posterior lateral intraparietal sulcus and the parieto-occipital region. Switching in RS was associated with activation in the medial intraparietal sulcus, adjacent posterior SPL and dorsomedial parietal cortex, and the anterior lateral intraparietal sulcus.

\section{Visual attention and visuomotor intention}

The first task, VS, involved attentional task switching. Subjects used a changing rule to select one of two visual stimuli for attention. The selection rule is based on either the color or shape of the stimuli. It is known that attentional selection for a the color or shape of a stimulus also entails spatial attention to its location (Tsal and Lavie, 1988, 1993). The notion of visual attention is a familiar one, and it is established that it is associated with the posterior parietal cortex (Corbetta et al., 1993, 1995, 2000; Fink et al., 1997a,b; Nobre et al., 1997; Coull and Nobre, 1998; Le et al., 1998; Corbetta and Shulman, 1999; Gitelman et al., 1999; Hopfinger et al., 2000; Macaluso et al., 2000; Vandenberghe et al., 2000). Different studies, however, have emphasized the importance of either inferior, superior, or even medial parietal cortex or all these areas. In the present study only a limited region was activated that was clearly ventral to the posterior parietal RS activation, suggesting a location in the LIP. Nobre et al. (1996, 1997) and Corbetta et al. (1993, 1995, 2000) were able to determine that the core visuospatial attention region is within the intraparietal sulcus, although they were unable to ascertain its position relative to limb movement areas. The limited extent of activation in the VS experiment may reflect the limited use of manual responses by subjects; subjects only made overt responses on $20 \%$ of trials, so the VS results are unlikely to reflect attentional modulation of response-related processes. The RS results suggest that attentional modulation in the SPL and medial parietal cortex is related to manual response components.

The second paradigm, RS, involved intentional task-switching. Switching involved changing the rules for movement selection rather than changing the rules for stimulus selection. Although the experimental manipulation used in RS is distinct to that used in many sensory attentional experiments, it is clear that the switch-stay activation in RS is the consequence of the operation of attention; on average, subjects saw the same stimuli and made the same responses during the switch and stay epochs, the only difference was the task-switching context in which stimuli and responses occurred. There was little overlap between the parietal areas activated on switching in RS and VS. RS activations were more dorsal and medial and included the MIP and area PEp (Von Economo and Koskinas, 1925) in the posterior SPL and extending onto the adjacent dorsomedial surface. In addition, there were several foci in the anterior IPL, the supramarginal gyrus, and the adjacent AIP. In comparison, only one activation was recorded in this region in the VS task.

The notion of motor-related attention, or intention (Boussaoud and Wise, 1993a,b), is less familiar than that of visual attention. It is, however, conceivable that there are covert intentional processes that are related to overt limb movements in much the same way that covert visual attention and orienting are related to overt eye movements (Sheliga et al., 1994; Snyder et al., 1997; Colby and Goldberg, 1999). It has previously been shown that intention depends on the supramarginal gyrus and AIP; these areas are activated more when subjects covertly attend to or prepare movements (Deiber et al., 1996; Krams et al., 1998; Rushworth et al., 2001b). Lesions and TMS inactivation in this region prevent subjects from switching attention from one movement to another (Rushwoth et al., 1997, 2001a). The present results demonstrate complementary specializations in LIP and AIP for visual attention and visuomotor intention. The complementary specializa- 

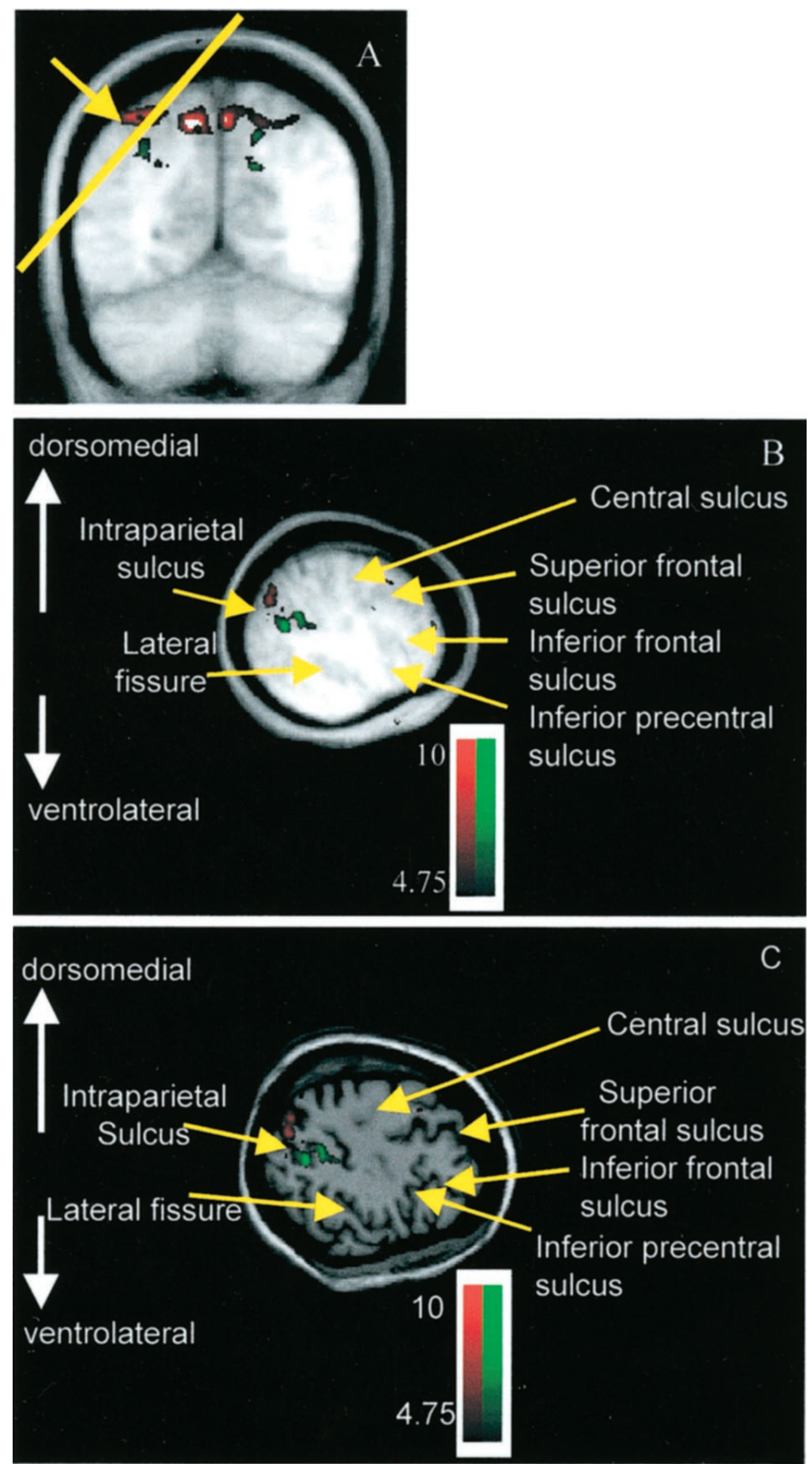

Figure 7. The position of the VS ( green) and RS (red) activations either side of the intraparietal sulcus can be best appreciated in a section orthogonal to the main axis of the intraparietal sulcus. $A$ shows a coronal section taken through the parietal lobe. The position of the intraparietal sulcus is indicated with an arrow. The yellow line shows the angle of a plane of section approximately orthogonal to the intraparietal sulcus. $B$ shows the section orthogonal to the intraparietal sulcus on the group average MRI. Within the posterior intraparietal sulcus RS activations, in the medial bank of the intraparietal sulcus and the posterior SPL, are more caudal, dorsal, and medial. VS activations in the posterior intraparietal sulcus are more rostral, ventral, and lateral, in the lateral bank of the intraparietal sulcus. $C$ shows the same activations on the anatomical MRI scan of a single representative subject.

tions of LIP and AIP may underlie their activation in overt eye and hand movement tasks, respectively (Kawashima et al., 1996, De Souza et al., 2000).

The more posterior superior parietal areas, MIP and PEp, have not been conspicuously implicated in previous studies of covert motor intention. Such studies simply required subjects to attend to an upcoming movement or to switch attention from one

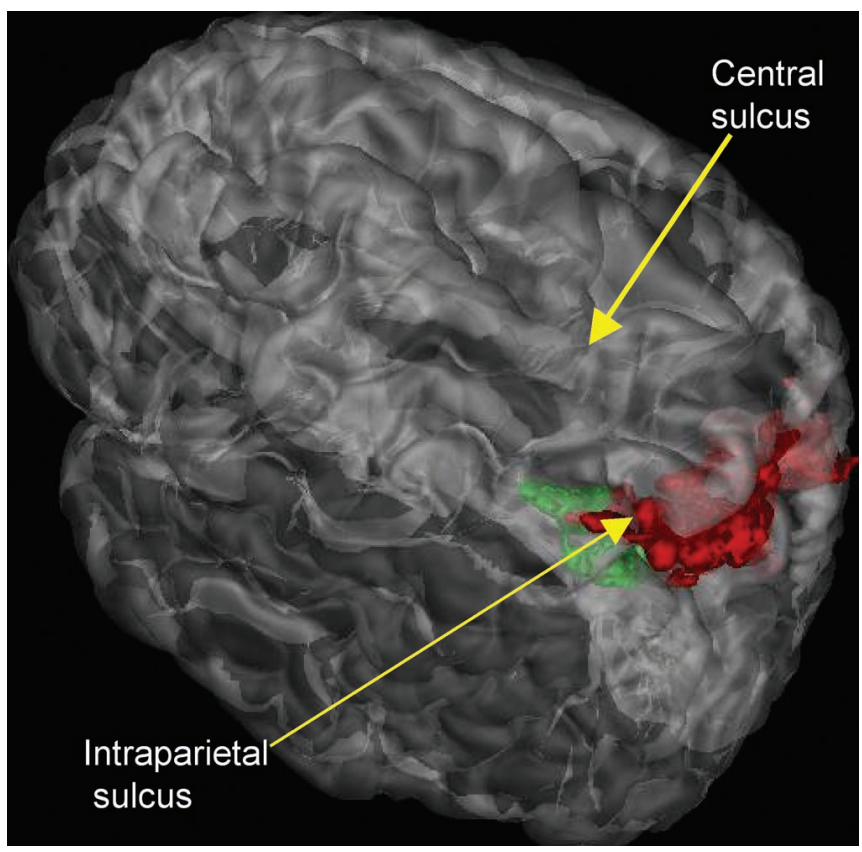

Figure 8. The more ventral, lateral, and rostral position of VS ( green) activations in the posterior parietal cortex, compared with RS (red) activations, can be seen in a 3-D view of semi-transparent brain anatomy.

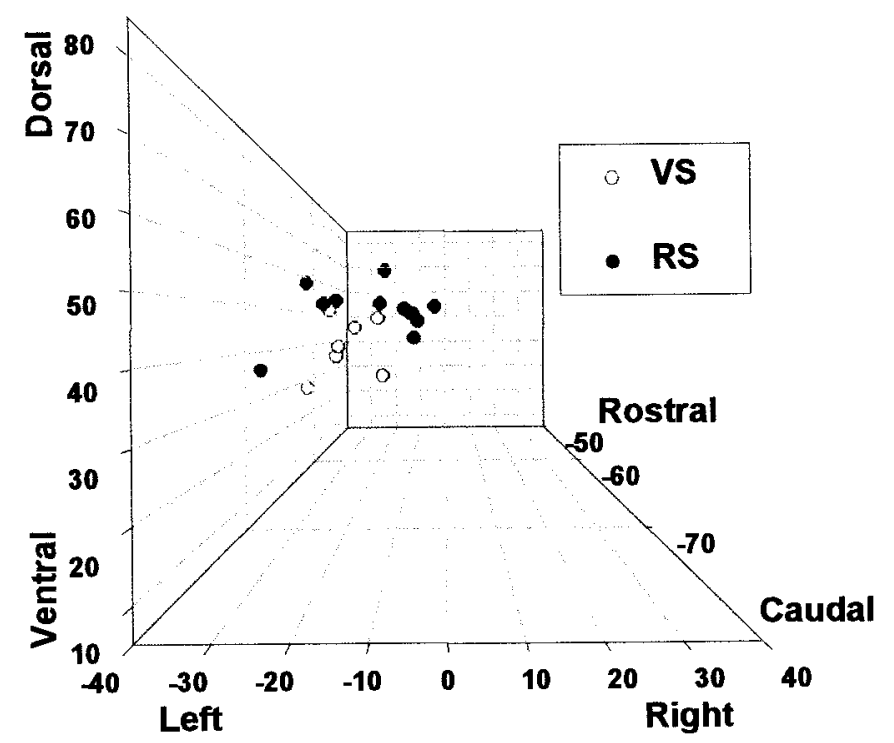

Figure 9. The positions of posterior parietal activations recorded in VS (O) and RS (O) tasks have been plotted on a 3-D graph. Although this mode of presentation tends to underemphasize rostrocaudal differences, the distinct localization of VS and RS activations is clear. All activations have been plotted as if they fell into the left hemisphere. The VS-RS separation is not, therefore, a spurious consequence of activations being lateralized to different hemispheres.

movement to another. The RS task, however, is more complex; it requires a switch from one visuomotor transformation to another, and MIP and PEp may be critical for this distinct attentional process. Other studies have considered a role for posterior parietal cortex in learning visuomotor rules, the production of spatially incompatible responses, or visually guided reaching (Grafton et al., 1992, 1996; Clower et al., 1996; Iacoboni et al., 


\begin{tabular}{|c|c|c|c|c|}
\hline & \multicolumn{2}{|l|}{ RS } & \multicolumn{2}{|l|}{ VS } \\
\hline & Talairach coordinates & $t$ values & Talairach coordinates & $t$ values \\
\hline \multirow[t]{2}{*}{ Parieto-occipital region (PO) } & & & (R) $15-6634$ & 8.86 \\
\hline & & & (R) $29-6933$ & 5.90 \\
\hline \multirow[t]{4}{*}{ Posterior lateral intraparietal sulcus (LIP) } & (L) $-33-7238$ & 6.19 & (L) $-34-5937$ & 10.84 \\
\hline & & & (L) $-33-5446$ & 9.15 \\
\hline & & & (L) $-19-7242$ & 6.32 \\
\hline & & & (R) $16-6648$ & 10.27 \\
\hline \multirow[t]{2}{*}{ Medial intraparietal sulcus (MIP) } & (L) $-23-7150$ & 7.47 & (L) $-23-7049$ & 5.34 \\
\hline & (L) $-33-6054$ & 12.91 & & \\
\hline \multirow[t]{3}{*}{ Posterior SPL (PEp) } & $(\mathrm{R}) * 17-6452$ & 7.15 & & \\
\hline & (L) $-29-6955$ & 11.21 & & \\
\hline & (R) $10-7355$ & 8.22 & & \\
\hline \multirow[t]{5}{*}{ Posterior SPL (medial) (PEp) } & (L) $-11-6351$ & 14.40 & (L) $-10-6250$ & 5.48 \\
\hline & (L) $-6-7148$ & 10.89 & & \\
\hline & (L) $-4-7744$ & 7.79 & & \\
\hline & (R) $3-6152$ & 12.59 & & \\
\hline & (R) $9-5748$ & 8.86 & & \\
\hline Anterior intraparietal sulcus (AIP) & (L) $-48-4148$ & 11.31 & (L) $-45-3438$ & 7.45 \\
\hline \multirow[t]{4}{*}{ Supramarginal gyrus } & (L) $-53-3443$ & 18.78 & & \\
\hline & (L) $-38-4439$ & 7.79 & & \\
\hline & (R) $50-3530$ & 5.97 & & \\
\hline & (R) $29-4045$ & 5.55 & & \\
\hline
\end{tabular}

$t>4.75, p<0.01 ; t>5.19, p<0.001$.

*Near to region assigned to the lateral bank of the sulcus.

Table 2. Parietal cortex activations identified in between task comparison

\begin{tabular}{|c|c|c|c|c|}
\hline & \multicolumn{2}{|l|}{$\mathrm{RS}-\mathrm{VS}$} & \multicolumn{2}{|l|}{ VS-RS } \\
\hline & Talairach coordinates & $t$ values & Talairach coordinates & $t$ values \\
\hline \multirow[t]{3}{*}{ Posterior lateral intraparietal sulcus (LIP) } & & & (L) $-26-6633$ & 3.43 \\
\hline & & & (L) $-29-4844$ & 3.74 \\
\hline & & & (L) $-30-4554$ & 6.56 \\
\hline \multirow[t]{2}{*}{ Medial intraparietal sulcus (MIP) } & (L) $-17-7853$ & 3.78 & & \\
\hline & (L) $-26-7947$ & 4.41 & & \\
\hline Posterior SPL (PEp) & (L) $-29-7546$ & 3.80 & & \\
\hline Anterior intraparietal sulcus (AIP) & (L) $-53-3825$ & 5.35 & & \\
\hline
\end{tabular}

Supramarginal gyrus

$t>3.42 ; p<0.01$.

1996; Deiber et al., 1997; Faillenot et al., 1997; Honda et al., 1998). However, as with the case of visual attention, there has been disagreement about the precise region that is critical. Moreover, there has been confusion about whether activations recorded in such tasks are the consequence of their visual attentional demands or, vice versa, whether visual attention tasks manipulate visuomotor response factors. The current results demonstrate an anatomical distinction between both processes.

Wise et al. (1997a) have discussed how parietal cortex, in conjunction with premotor cortex, may be important for learned visuomotor transformations, although its precise role has been uncertain (Halsband and Passingham, 1982; Rushworth et al., 1997a). It seems clear that frontal lobe areas are more important in the learning of such transformations (Wise and Murray, 2000). The current results suggest one role of parietal areas MIP and PEp may be intentional switches between visuomotor transformation rules.

\section{The organization of the parietal lobe in monkeys and people}

The distribution of VS- and RS-related areas around the intraparietal sulcus suggest that the human intraparietal sulcus and the surrounding parietal cortex has a similar organization to that of the macaque monkey.

The VS task was associated with modulation in human LIP and PO. Areas LIP and PO occupy similar locations in posterior lateral intraparietal and parieto-occipital cortex in the macaque and contain visually responsive cells that are important in visual attention and oculomotor control (Galletti et al., 1993, 1995; Andersen et al., 1998; Colby and Goldberg, 1999).

Cells on the posterior MIP of the macaque have distinct properties; they also respond to visual stimuli, but their activity is related more closely to limb movements than eye movements (Colby and Duhamel, 1991; Snyder et al., 1997; Eskander and Assad, 1999). Similar visuomotor activity has also been found in 
adjacent tissue in the most posterior SPL, the mediodorsal parietal cortex, and the most dorsal parieto-occipital cortices (PEc, MDP, V6A) (Caminiti et al., 1996; Galletti et al., 1997; BattagliaMayer et al., 2000; Ferraina et al., 2001). Whether each of these areas of the macaque brain have distinct functions remains to be clarified. The same human areas, MIP and PEp, medial and dorsal to those concerned with visual attention, were activated in RS.

The macaque AIP and adjacent anterior IPL (PF) also have some visually responsive cells and are related to limb, perhaps particularly hand, movements (Sakata et al., 1999). Similar areas in the human parietal cortex were also activated in the RS task.

In summary, the results are consistent with a scheme in which the intraparietal sulcus divides the parietal cortices in a similar way in both monkeys and people (Von Bonin and Bailey, 1947; Eidelburg and Galaburda, 1984) rather than with the currently more widely used scheme in which the human IPL is regarded as a novel structure (Brodmann, 1909). Functional areas appear to occupy similar positions relative to one another and to the main anatomical landmark in the area, the intraparietal sulcus. In accordance with this scheme, Bremmer et al. (2001) have recently presented functional data showing that the human VIP, like macaque VIP, is in the depths of the intraparietal sulcus. Crossspecies anatomical correspondences in the temporal and frontal lobes have been difficult to establish (Petrides and Pandya, 1994; Preuss, 1995), but the organization of the parietal cortex may have been conserved during primate speciation. Preuss and Goldman-Rakic (1991) have demonstrated that the parietal lobe, including the intraparietal sulcus, in the strepsirhine Galago has a similar organization to that in the anthropoid macaque. Different parietal areas are concerned with distinct attentional processes and transformations between incoming sensory information and movements of either the eye or the limb. The types of sensorimotor transformations and related attentional processes required by primates may have remained stable since the development of binocular vision and reaching and grasping hand and arm movements (Sakata et al., 1997). Because parietal areas are concerned with attention and sensorimotor transformations, the basic plan for parietal organization may have been conserved from strepsirhines to humans.

\section{REFERENCES}

Allport DA, Styles EA, Hsieh S (1994) Shifting intentional set: exploring the dynamic control of tasks. In: Attention and performance, Vol 15 (Umilta C, Moscovitch M, eds), pp 421-452. Cambridge, MA: MIT.

Andersen RA, Snyder LH, Batista AP, Buneo CA, Cohen YE (1998) Posterior parietal areas specialized for eye movements (LIP) and reach (PRR) using a common coordinate frame. Novartis Found Symp 218:109-122.

Battaglia-Mayer A, Ferraina S, Mitsuda T, Marconi B, Genovesio A, Onorati P, Lacquaniti F, Caminiti R (2000) Early coding of reaching in the parietooccipital cortex. J Neurophysiol 83:2374-2391.

Boussaoud D, Wise SP (1993a) Primate frontal cortex: neuronal activity following attentional versus intentional cues. Exp Brain Res 95:15-27.

Boussaoud D, Wise SP (1993b) Primate frontal cortex: effects of stimulus and movement. Exp Brain Res 95:28-40.

Bremmer F, Schlack A, Shah NJ, Zafiris O, Kubischik M, Hoffmann K, Zilles K, Fink GR (2001) Polymodal motion processing in posterior parietal and premotor cortex: a human fMRI study strongly implies equivalencies between humans and monkeys. Neuron 29:287-296.

Brodmann K (1909) Vergleichende Lokalisationslehre der Grosshirnrinde in ihren Prinzipien dargestellt auf Grund des Zellenbaues. Liepzig: Barth (translated by L. J. Garey (1994), Brodmann's "Localization in the cerebral cortex", London: Smith-Gordon).

Caminiti R, Ferraina S, Johnson PB (1996) The sources of visual information to the primate frontal lobe: a novel role for the superior parietal lobule. Cereb Cortex 6:319-328.

Colby CL, Duhamel J-R (1991) Heterogeneity of extrastriate visual areas and multiple parietal areas in the macaque monkey. Neuropsychologia 29:517-537.
Colby CL, Goldberg ME (1999) Space and attention in parietal cortex. Annu Rev Neurosci 22:319-349.

Collins DL, Neelin P, Peters TM, Evans AC (1994) Automatic 3D intersubject registration of MR volumetric data in standardized Talairach space. J Comput Assist Tomogr 18:192-205.

Corbetta M, Shulman GL (1999) Human cortical mechanisms of visual attention during orienting and search. In: Attention, space, and action: studies in cognitive neuroscience (Humphreys GW, Duncan J, and Triesman A, eds). Oxford: OUP.

Corbetta M, Miezin FM, Shulman GL, Petersen SEA (1993) A PET study of visuospatial attention. J Neurosci 13:1202-1226.

Corbetta M, Shulman GL, Miezin FM, Petersen SE (1995) Superior parietal cortex activation during spatial attention shifts and visual feature conjunction. Science 270:802-804.

Corbetta M, Kincade JM, Ollinger JM, McAvoy MP, Shulman GL (2000) Voluntary orienting is dissociated from target detection in human posterior parietal cortex. Nat Neurosci 3:292-297.

Coull JT, Nobre AC (1998) Where and when to pay attention: the neural substrate for directing attention to spatial locations and to time intervals as revealed by both PET and fMRI. J Neurosci 18:7426-7435.

Deiber M-P, Ibanez V, Sadato N, Hallet M (1996) Cerebral structures participating in motor preparation in humans: a positron emission tomography study. J Neurophysiol 75:233-247.

Deiber M-P, Wise SP, Honda M, Catalan MJ, Grafman J, Hallett M (1997) Frontal and parietal networks for conditional motor learning: a positron emission tomography study. J Neurophysiol 78:977-991.

De Souza JFX, Dukelow SP, Gati JS, Menon RS, Andersen RA, Vilis T (2000) Eye position signal modulates a human parietal pointing region during memory-guided movements. J Neurosci 20:5835-5840.

Eidelburg D, Galaburda AM (1984) Inferior parietal lobule: divergent architectonic asymmetries in the human brain. Arch Neurol 41:843-852.

Eskander EN, Assad JA (1999) Dissociation of visual, motor and predictive signals in parietal cortex during visual guidance. Nat Neurosci 2:88-93.

Evans AC, Collins DL, Milner B (1992) An MRI-based stereotaxic atlas from 250 young normal subjects. Soc Neurosci Abstr 18:408.

Faillenot I, Toni I, Decety J, Gregoire M-C, Jeannerod M (1997) Visual pathways for object-oriented action and object recognition: functional anatomy with PET. Cereb Cortex 7:77-85.

Ferraina S, Battaglia-Mayer A, Genovesio A, Marconi B, Onorati P, Caminiti R (2001) Early coding of visuomanual coordination during reaching in parietal area PEc. J Neurophysiol 85:462-467.

Fink GR, Dolan RJ, Halligan PW, Marshall JC, Frith CD (1997a) Spacebased and object-based visual attention: shared and specific neural domains. Brain 120:2013-2028.

Fink GR, Halligan PW, Marshall JC, Frith CD, Frackowiak RSJ, Dolan RJ (1997b) Neural mechanisms involved in the processing of global and local aspects of hierarchically organized visual stimuli. Brain 120:1779-1791.

Galletti C, Battaglini PP, Fattori P (1993) Parietal neurons encoding spatial locations in craniotopic coordinates. Exp Brain Res 96:221-229.

Galletti C, Battaglini PP, Fattori P (1995) Eye position influence on the parieto-occipital area PO (V6) of the macaque monkey. Eur J Neurosci 7:2486-2501.

Galletti C, Fattori R, Kutz DF, Battaglini PP (1997) Arm movement related neurons in the visual area $\mathrm{V} 6$ of the macaque superior parietal lobule. Eur J Neurosci 9:410-413.

Gitelman DR, Nobre AC, Parrish TB, LaBar KS, Kim YH, Meyer JR, Mesulam M (1999) A large scale distributed network for covert spatial attention: further anatomical delineation based on stringent behavioural and cognitive controls. Brain 122:1093-1106.

Grafton ST, Mazziota JC, Woods RP, Phelps ME (1992) Human functional anatomy of visually guided finger movements. Brain 115:565-587.

Grafton ST, Fagg AH, Woods RP, Arbib MA (1996) Functional anatomy of pointing and grasping. Cereb Cortex 6:226-237.

Halsband U, Passingham RE (1982) The role of premotor and parietal cortex in the direction of action. Brain Res 240:368-372.

Honda M, Wise SP, Weeks RA, Deiber M-P, Hallett M (1998) Cortical areas with enhanced activation during object centred spatial information processing: a PET study. Brain 121:2145-2158.

Hopfinger JB, Buonocore MH, Mangun GR (2000) The neural mechanisms of top-down attentional control. Nat Neurosci 3:284-291.

Iacoboni M, Woods RP, Mazziotta JC (1996) Brain behaviour relationships: evidence from practise effects in spatial stimulus-response compatibility. J Neurophysiol 76:321-331.

Kawashima R, Naitoh E, Matsumura M, Itoh H, Ono S, Satoh K, Gotoh R, Koyama M, Inoue K, Yoshioka S, Fukuda H (1996) Topographic representation in human intraparietal sulcus of reaching and saccade. NeuroReport 7:1253-1256.

Krams M, Rushworth MFS, Deiber MP, Frackowiak RSJ, Passingham RE (1998) The preparation, suppression, and execution of copied movements in the human brain. Exp Brain Res 120:386-398.

Le TH, Pardo JV, Hu X (1998) 4 T-fMRI study of nonspatial shifting of 
selective attention: cerebellar and parietal contributions. J Neurophysiol 79:1535-1548.

Macaluso E, Frith CD, Driver J (2000) Modulation of human visual cortex by crossmodal spatial attention. Science 289:1206-1208.

Nobre AC, Sebestyen GN, Gitelman DR, Mesulam MM, Frackowiak RSJ, Frith CD (1997) Functional localization of the system for visuospatial attention using position emission tomography. Brain 120: $515-533$.

Petrides M, Pandya DN (1994) Comparative architectonic analysis of the human and the macaque frontal cortex. In: Handbook of neuropsychology, Vol 9 (Boller F and Grafman J, eds), pp 17-58. Amsterdam: Elsevier.

Preuss TM (1995) Do rats have prefrontal cortex? The Rose-WoolseyAkert program reconsidered. J Comp Neurol 7:1-24.

Preuss TM, Goldman-Rakic PS (1991) Architectonics of the parietal and temporal association cortex in the strepsirhine Galago compared to the anthropoid primate Macaca. J Comp Neurol 310:475-506.

Rogers RD, Monsell S (1995) The cost of a predictable switch between simple cognitive tasks. J Exp Psychol Gen 124:207-231.

Rushworth MFS, Nixon PD, Passingham RE (1997a) The parietal cortex and movement. I. Movement selection and reaching. Exp Brain Res 117:292-310.

Rushworth MFS, Nixon PD, Renowden S, Wade DT, Passingham RE (1997b) The left parietal cortex and attention to action. Neuropsychologia 35:1261-1273.

Rushworth MFS, Hadland KA, Passingham RE, Nobre AC (1999) Redirecting motor attention and the pre-SMA: rTMS and ERP recording. Soc Neurosci Abstr 865.14.

Rushworth MFS, Ellison A, Walsh V (2001a) Complementary localization and laterization of orienting and motor attention. Nat Neurosci 4:656-661.

Rushworth MFS, Krams M, Passingham RE (2001b) Complementary lateralization of attention and intention in the human brain. A PET study. J Cogn Neurosci, in press.

Sakata H, Taira M, Kusunoki M, Murata A, Tanaka Y (1997) The TINS lecture. The parietal association cortex in depth perception and visual control of hand action. Trends Neurosci 20:350-357.

Sakata H, Taira M, Kusunoki M, Murata A, Tsutsui K, Tanaka Y, Shein WN, Miyashita Y (1999) Neural representation of three dimensional features of manipulation objects with stereopsis. Exp Brain Res 128:160-169

Sheliga BM, Riggio L, Rizzolatti G (1994) Orienting of attention and eye movements. Exp Brain Res 98:507-522.

Snyder LH, Batista AP, Andersen RA (1997) Coding of intention in the posterior parietal cortex. Nature 386:167-170.

Snyder LH, Batista AP, Andersen RA (1998) Change in motor plan, without a change in the spatial locus of attention, modulates activity in posterior parietal cortex. J Neurophysiol 79:2814-2819.

Talairach J, Tournoux P (1988) Co-planar stereotaxic atlas of the human brain. Thieme: Stuttgart.

Tsal Y, Lavie N (1988) Attending to colour and shape: the special role of location in selective visual processing. Percept Psychophys 44:15-21.

Tsal Y, Lavie N (1993) Location dominance in attending to colour and shape. J Exp Psychol Hum Percept Perform 19:131-139.

Vandenberghe R, Duncan J, Arnell KM, Bishop SJ, Herrod NJ, Owen AM, Minhas PS, Dupont P, Pickard JD, Orban GA (2000) Maintaining and shifting attention within left or right hemifield. Cereb Cortex 10:706-713.

Von Bonin G, Bailey P (1947) The neocortex of Macaca mulatta. Urbana, IL: University of Illinois.

Von Economo C (1929) The cytoarchitectonics of the human cerebral cortex. London: Oxford UP.

Von Economo C, Koskinas GN (1925) Die Cytoarchitecktonik der Hirnrinde des erwachsenen Menschen. Berlin: Springer.

Wise SP, Murray EA (2000) Arbitrary associations between antecedents and actions. Trends Neurosci 23:271-276.

Wise SP, Boussaoud D, Johnson PB, Caminiti R (1997) Premotor and parietal cortex: corticortical connectivity and combinatorial computations. Annu Rev Neurosci 20:25-42.

Worsley KJ, Marrett S, Neelin P, Vandal AC, Friston KJ, Evans AC (1996) A unified statistical approach for determining significant signals in images of cerebral activation. Hum Brain Mapp 4:58-73.

Worsley KJ, Andermann M, Koulis T, MacDonald D, Evans AC (1999) Detecting changes in nonisotropic images. Hum Brain Mapp 8:98-101. Worsley KJ, Liao C, Grabove M, Petre V, Ha B, Evans AC (2000) A general statistical analysis for fMRI data. NeuroImage 10:S648.

Zarahn E, Aguirre G, D'Esposito M (1997) A trial-based experimental design for fMRI. NeuroImage 6:122-138. 